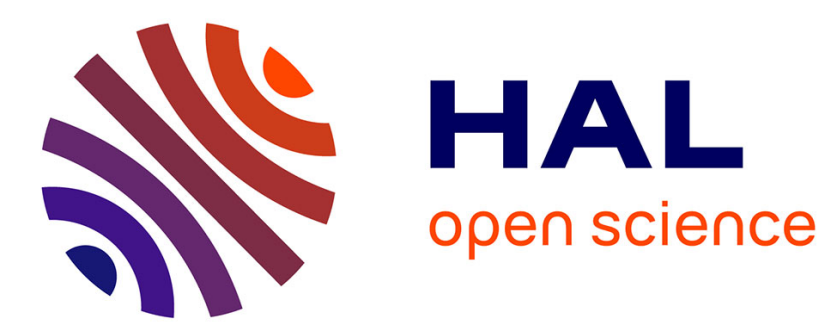

\title{
MODĖLES DE TRANSMISSION VIBRATOIRE PAR LES COUPLAGES SURFACIQUES
}

S. Naji, C. Cacciolati, Jean-Louis Guyader

\section{To cite this version:}

S. Naji, C. Cacciolati, Jean-Louis Guyader. MODÈLES DE TRANSMISSION VIBRATOIRE PAR LES COUPLAGES SURFACIQUES. Journal de Physique IV Proceedings, 1992, 02 (C1), pp.C1-519C1-522. 10.1051/jp4:19921112 . jpa-00251067

\section{HAL Id: jpa-00251067 https://hal.science/jpa-00251067}

Submitted on 1 Jan 1992

HAL is a multi-disciplinary open access archive for the deposit and dissemination of scientific research documents, whether they are published or not. The documents may come from teaching and research institutions in France or abroad, or from public or private research centers.
L'archive ouverte pluridisciplinaire HAL, est destinée au dépôt et à la diffusion de documents scientifiques de niveau recherche, publiés ou non, émanant des établissements d'enseignement et de recherche français ou étrangers, des laboratoires publics ou privés. 


\title{
MODẼLES DE TRANSMISSION VIBRATOIRE PAR LES COUPLAGES SURFACIQUES
}

\author{
S. NAJI, C. CACCIOLATI et J.L. GUYADER \\ LVA, Bât 303, INSA, 20 Av Einstein, F-69621 Villeurbanne cedex, France
}

\begin{abstract}
RESUME: Nous étudions un assemblage de deux structures de densité modale trés différentes couplées surfaciquement. Le schéma modal des structures est approché en utilisant trois données expérimentales mesurée avant couplage. La prévision de la vitesse couplée est en accord avec l'expérience.
\end{abstract}

SUMMARY: A semi anlytical method to study the behaviour of the vibration of coupled structures is presented. The coupling area is the whole surface of one of the elements. Three measurements are done in order to define approximately the modal bases. Theory and experiment are in good agreement.

\section{Introduction}

L'étude vibroacoustique des assemblages a été traitée avec succès dans le cas des couplages ponctuels par LYON [1] et récemment par U. CARLSSON [2] et S. NAJI [3]. La méthode devient lourde pour les couplages linéïques J.M. CUSCHEIRI [4] et nécessite des simplifications extrêmes pour les couplages surfaciques B. PETERSSON J. PLUNT [5]. On observe expérimentalement que pour une structure de forte densité modale le spectre fréquentiel de la mobilité d'entrée est en première approximation identique en tout point. Pour le spectre de la mobilité de transfert entre deux points $M$ et $N$, le module est peu dépendant de $M$ et $N$ alors que la phase varie. Nous présentons une méthode semi analytique pour traiter les couplages surfaciques correctement avec des calculs numériques légers. $\mathrm{Ce}$ travail est réalisé avec l'aide et la collaboration du Ministère de l'Environnement et du Cadre de Vie (S.R.E.T.I.) et de la Compagnie Industrielle d'Appareillage Electro-ménager (CIAPEM).

\section{Modélisation d'un couplage surfacique}

Notre modèle d'étude est constitué par une plaque support mince, à forte densité modale. Une ou plusieurs plaques rapportées à faible densité modale sont couplées sur toute leur surface avec

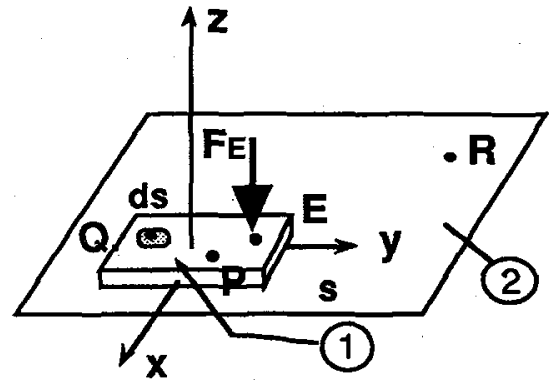
la plaque support. En tout points de la surface de couplage $s$ nous supposons que les efforts de couplage sont réduits à une force surfacique normale à $s$. Sur chaque élément de surface ds nous exprimons la continuité des vitesses $V 1$ et $V 2$ et l'équilibre des forces de couplage FC. L'équation d'équilibre du système couplé s'exprime en fonction des mobilités de transfert de chaque élément, des efforts de couplage FC et de la force d'excitation ponctuelle FE

\section{FIGURE No1}




$$
\int_{S}\left[M 1_{(P, Q)}+M 2_{(P, Q)}\right] F C_{(Q)} d s_{Q}=M 2_{(Q, E)} F E
$$

Pour obtenir la loi de répartition spatiale $F C(Q)$ des forces de couplage nous projetons cette relation sur la base tronquée des $\mathbf{N}$ premiers modes de la plaque rapportée. Les modes de cette plaque sont définis par les fréquences propres $f_{i}$ mesurées et par les formes propres $\Phi \mathbf{i}$ approchées par les formes propres de poutre libre-libre d'après BLEVINS [6]. Les trois premiers modes solides de pulsation propre nulle sont considérés, ainsi que les premiers modes de flexion. Après projection nous obtenons pour tout mode d'ordre $\mathrm{k}$ l'équation suivante.

$$
\sum_{i=1}^{N} F C_{(i)} \int_{S}\left[\int_{S} M 1_{(P, Q)} \varnothing_{i(Q)} d s_{Q}\right] \varnothing_{k(P)} d s p+M 2_{k} F C_{k} N_{k}^{2}=M 2_{k} N_{k}^{2} \varnothing_{k(E)} F E
$$

$$
\begin{array}{l|l}
F C_{(P)}=\sum_{k=1}^{N} F C_{k} \varnothing_{k}(P) & M 2_{k}=\sum_{k=1}^{N} \frac{j \omega}{m_{k}\left[\left(\omega_{k}^{2}-\omega^{2}\right)+2 j \omega_{k} \omega \eta_{k}\right]} \\
M 2_{(P, Q)}=\sum_{k=1}^{N} M 2_{k} \varnothing_{k}(P) \varnothing_{k}(P) & m_{k}=\rho h \int_{S} \varnothing_{k}^{2} d s=\rho h N_{k}
\end{array}
$$

Le système d'équations à résoudre est:

$$
\left[\left[A_{k i}\right]+\left[B_{k i}\right]\right]\left[C_{i}\right]=\left[\widetilde{V_{k}}\right]
$$

avec:

$$
\begin{aligned}
& A_{k i}=\int_{s}\left[\int_{s} M 1(P, Q) \varnothing_{k}(P) d s p\right] \varnothing_{i}(Q) d s Q \\
& B_{k i}=M 2_{k} N_{k}^{2} \delta(i-k) \quad \text { et } \quad \tilde{V}_{k}=M 2_{k} N_{k} \varnothing_{k(E)} F E
\end{aligned}
$$

La matrice [A] est pleine, elle représente les couplages intermodaux provoqués par la présence de la plaque support. La matrice [B] est diagonale, elle traduit la contribution de la plaque rapportée. Le vecteur [V] représente les composantes modales de la vitesse de la plaque rapportêe excitée par $F E$ et non couplée à la plaque support, c'est la vitesse avant couplage. La vitesse en un point $P$ de $s$, la vitesse quadratique moyenne sur $s$ et la puissance échangée sont:

$$
\begin{array}{cc}
V_{(P)}=\sum_{k=1}^{N}\left[V_{k}\right]^{T}\left[\varnothing_{k}(P)\right] \quad \text { avec } & {\left[V_{k}\right]=\left[\frac{1}{N}\right]\left[A_{k i}\right]\left[F C_{i}\right]} \\
\left.\left\langle\bar{V}^{2}\right\rangle=\frac{1}{2 s} \sum_{k=1}^{N} \frac{1}{N_{k}}\left(\sum_{i, L} A_{k i} A_{k L}^{*} F C_{i} F C_{L}^{*}\right) \quad \text { et } \quad \text { Puiss }=\frac{1}{2} R^{*} \operatorname{Rel}_{\left\{\sum_{i, L} F C_{i}^{*} A_{i} L\right.} F C_{L}\right\}
\end{array}
$$




\section{Modèles de mobilité pour la plaque support.}

Nous avons retenu que le module de la mobilité de la plaque support est peu dépendante de l'éloignement du point d'excitation et du point d'écoute. La figure 2, tirée de l'expérience montre les modules des mobilités directe au point $P$ et de transfert entre $P$ et $Q$ (voir figure $N^{\circ} 2$ ). Sur la base de ce constat nous étudierons quatre modèles pour représenter le transfert $M(P, Q)$.

Le modèle (a) majore l'influence de la mobilité de transfert. II considère qu'à chaque fréquence toutes les mobilités ont même phase: $M 1(P, Q)=$ Cte. On mesurera cette valeur sur la plaque support en un point arbitraire de s avant couplage. Le déplacement en tout point d'écoute est identique à celui du point excité. L'influence de la plaque support est assimilée à celle d'une masse rigide en translation.

Le modèle (b) utilise une mobilité delta correllée. II minore l'influence de la mobilité de transfert. Le déplacement du point d'écoute n'est fonction que de la mobilité d'entrée en ce point de la plaque support, les mobilités de transfert avec les autres points de s sont nulles: $M 1(P, Q)=M \delta(P$. $\mathrm{Q})$. On prendra pour $\mathrm{M}$ la valeur mesurée avant couplage sur la plaque support au centre de $\mathrm{s}$.

Le modèle $(c)$ est en cours d'étude, il utilise une phase propagative: $M 1=M$ e j K r $(P, Q)$ où $\mathrm{K}$ est le nombre d'onde de flexion d'une plaque infinie.

Le modèle (d) est également en cours d'étude. La mobilité d'entrée est identique en tout point de $s$, c'est une valeur $M$ mesurée au centre de $s$ avant couplage, elle représente l'effet du champ proche. La mobilité de transfert est inspirée du modèle de phase réverbérée décrite par LYON [7] on la suppose identique entre tout points $P, Q$ de s. Cette mobilité de transfert $M^{*}$ est mesurée entre deux points éloignés de la plaque support avant couplage, elle représente le champ réverbéré de la plaque support. La mobilité de la plaque support est $M 1=M^{*}+\left(M-M^{*}\right) e^{-\alpha} r(P, Q)$. La constante $\alpha$ représente l'étendue du champ proche.

\section{Comparaison Théorie - Expérience}

Nous utilisons une plaque d'acier de $1,5 \mathrm{~mm}$ d'epaisseur encastrée sur son pourtour, la plaque rapportée est simplement posée, elle tient par gravité et un film d'huile lubrifie le contact pour limiter au maximun les efforts tangentiels. L'huile introduit un amortissement supplémentaire. L'excitation est une force ponctuelle au centre de $\mathrm{s}$. La plaque rapportée à $1 \mathrm{~cm}$ d'épaisseur. La figure No2 compare les modules des mobilités d'entrée en $P$ et de transfert entre $P$ et $Q . p o u r$ la plaque support seule.
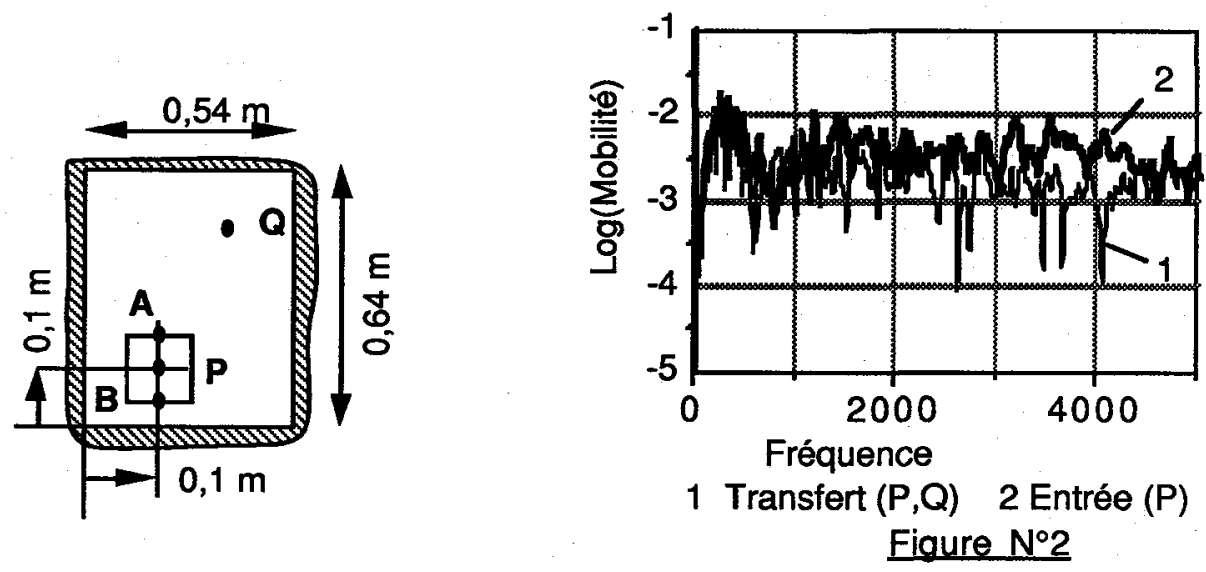
La figure $N^{\circ} 3$ compare le modèle (b) avec l'expérience pour la mobilité d'entrée après couplage en $P$, milieu d'une plaque rapportée de $0,25 \times 0,13 \times 0,01 \mathrm{~m}$ en acier. On constate que ce modèle est représentatif de la réalité. Le modèle (a), non représenté sur la figure, convient en basse fréquence avant le premier mode de la plaque rapportée, il représente la lois de masse $(-3 \mathrm{~dB} / \mathrm{oct})$ de la plaque rapportée. Sur la figure $N^{\circ} 4$ nous comparons les mobilités de transfert aprés couplage entre les points A rapportée. Sur la figure $\mathrm{N}^{\circ} 4$ nous comparons les mobilités de transfert après couplage entre les points $A$ et $B$ pour le modèle (b) et pour l'expérience avec une plaque rapportée de $0,1 \times 0,1 \times 0,01 \mathrm{~m}$ en acier. Nous constatons que c'est la plaque support qui contrôle le mouvement en basse fréquence. Dans tous les cas les modes des plaques rapportées pilotent la réponse à partir de leur première fréquence propre. La réponse résonante est limitée par la mobilité de la plaque support et par l'amortissement.

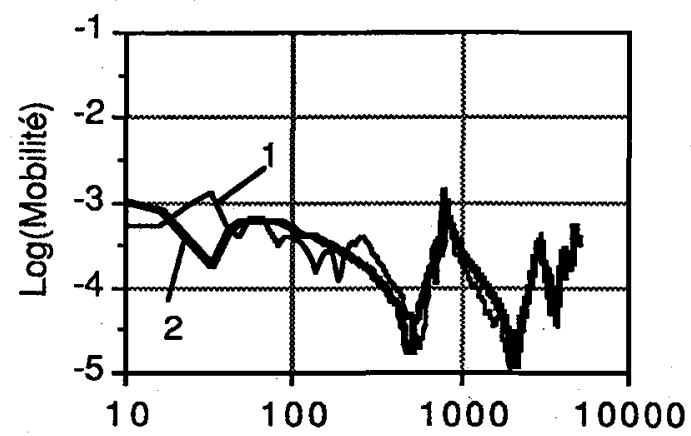

Fréquence

1 Expérience (P) 2 Calcul $(P)$

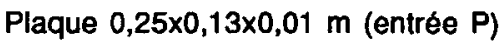
EIGURE No3

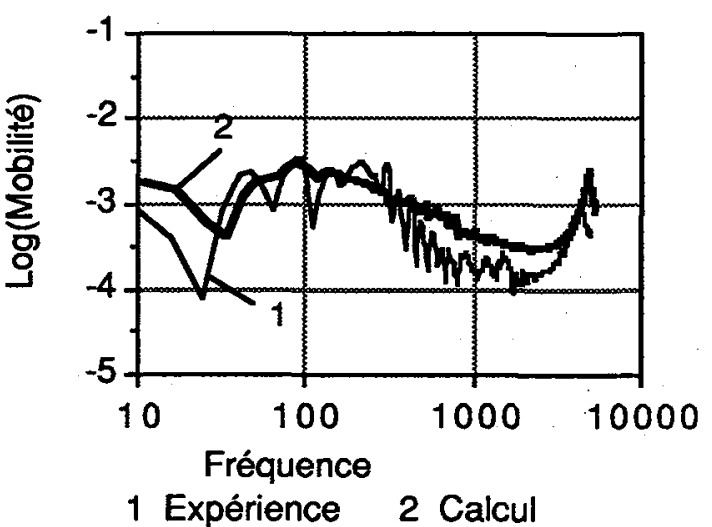

Plaque $0,1 \times 0,1 \times 0,01 \mathrm{~m}$ (Transfert $A, B$ ) EIGUREN4

\section{Conclusion}

La méthode semi analytique que nous proposons permet de traiter de façon satisfaisante le couplage surfacique dans les assemblages. Nous construisons les fonctions analytiques de mobilité en tout point des surfaces de couplage à partir de trois mesures. Deux spectres de mobilité mesurés sur la surface support (à forte densité modale), et un essai de choc sur la surface rapportée (de faible densité modale). La reconstruction du schéma modal de la structure rapportée s'est avérêe très satisfaisante. Nous n'avons mis en œuvre actuellement que deux des quatre modèles envisagés de mobilité pour la structure support. On constate que le modèle de mobilité delta-correllée, bien que très loin de la réalité est suffisant pour prévoir la réponse en tout point d'une surface de couplage quand elle reçoit une excitation directe. Mais ce modèle est incapable de prévoir la réponse sur une surface excitée par un transfert. Pour traiter ce cas, des modèles de phase propagative et de phase réverbérée sont en cours d'étude.

\section{Bibliographie}

(1) R.H. LYON. MIT Press. (1975)

(2) U. CARLSON Raport Novembre (1990)

(3) S. NAJI, Raport Decembre (1990)

(4)J.M. CUCHEIRI, JASA 87 (3) 1159-1165 (1990)

(5) B. PETERSSON, J. PLUNT, JSV 82 (4) $517-540$ (1982)

(6) Robert. D. BLEVINS, Robert E. krieger Publishing Company (1984)

(7) R. H. LYON J.A.S.A 76 (5) , 1433-1437 Novembre (1984) 